\title{
Gel Phase 1,2-Distearoyl-sn-glycero-3- phosphocholine-Based Liposomes Are Superior to Fluid Phase Liposomes at Augmenting Both Antigen Presentation on Major Histocompatibility Complex Class II and Costimulatory Molecule Display by Dendritic Cells in Vitro
}

\section{Supporting Information}

Karin Norling ${ }^{1}$, Valentina Bernasconi ${ }^{2}$, Víctor Agmo Hernández ${ }^{3,6}$, Nagma Parveen ${ }^{1}$, Katarina Edwards ${ }^{3}$, Nils Y. Lycke ${ }^{2}$, Fredrik Höök ${ }^{1 *}$, Marta Bally ${ }^{4,5 *}$

${ }^{1}$ Division of Biological Physics, Department of Physics, Chalmers University of Technology, 41296 Gothenburg, Sweden.

${ }^{2}$ Mucosal Immunobiology and Vaccine Center (MIVAC), Department of Microbiology and Immunology, Institute of Biomedicine, University of Gothenburg, 40530 Gothenburg, Sweden.

${ }^{3}$ Department of Chemistry - BMC, Uppsala University, Box 599, 75237 Uppsala, Sweden

${ }^{4}$ Section of Virology, Department of Clinical Microbiology, Umeå University, 901 85 Umeå, Sweden

${ }^{5}$ Wallenberg Centre for Molecular Medicine, Umeå University, 90185 Umeå, Sweden

${ }^{6}$ Department of Pharmacy, Uppsala University, Box 580, 751 23, Uppsala, Sweden

Number of Pages: 8

Number of Figures: 5

Number of Tables: 2 


\section{Section S1: Initial screening}

To systematically investigate the influence of physicochemical properties of vaccine vectors on antigen presentation, lipid particles of various compositions were produced (Table S1). Firstly, POPC-based liposomes, with and without poly-ethylene glycol (PEG) (abbreviated POPC-PEG-FP and POPC-PE-FP, respectively) were used for fusion protein delivery in order to investigate the influence of PEGylation on antigen presentation. POPC-PE-FP had the fusion protein coupled via maleimide groups directly to the phosphoethanolamine (PE) headgroups of DOPEMCC lipids, while in POPC-PEG-FP it was attached with the same type of chemistry to lipid-anchored PEG(2000) spacers on DSPEPEGMCC lipids. Secondly, to assess the influence of the number of attached fusion proteins per liposome, liposomes with high and low loads of fusion protein were used, (POPC-high-FP and POPC-low-FP, respectively). The protein load was tuned via the protein concentration used in the protein coupling step. Finally, to assess the influence of shape and size, the immunogenicity of DSPC-based lipodisks, flat, circular bilayer disks with an edge stabilized by PEGylated lipids ${ }^{1}$, were compared to spherical liposomes of a similar composition (abbreviated DSPC-PEGFP*). For both Lipodisks-FP and DSPC-PEG-FP*, the protein was attached via thiolmaleimide chemistry to the particles via PEG-spacers.

Table S1. Lipid compositions of the various particle variants. The lipids used were 1-palmitoyl-2-oleoyl-snglycero-3-phosphocholine (POPC), cholesterol, 1,2-dioleoyl-sn-glycero-3-phosphoethanolamine-N-[4-(pmaleimidomethyl)cyclohexane-carboxamide] phosphoethanolamine- $\mathrm{N}$-[maleimide(polyethylene glycero-3-phosphocholine (DSPC) and (DOPEMCC), 1,2-distearoyl-sn-glycero-31,2-distearoyl-sn-glycero-3-phosphoethanolamine-N[methoxy(polyethylene glycol)-2000] (ammonium salt) (DSPEPEG).

\begin{tabular}{|c|c|c|}
\hline $\begin{array}{l}\text { FORMULATIO } \\
\mathbf{N}\end{array}$ & LIPIDS & MOLAR RATIO \\
\hline POPC-PE-FP & POPC, cholesterol, DOPEMCC & $85: 10: 5$ \\
\hline POPC-PEG-FP & POPC, cholesterol, DSPEPEGMCC & $89: 10: 1$ \\
\hline POPC-HIGH-FP & POPC, cholesterol, DOPEMCC & $89: 10: 1$ \\
\hline POPC-LOW-FP & POPC, cholesterol, DOPEMCC & $89: 10: 1$ \\
\hline DSPC-PEG-FP* & DSPC, DSPEPEGMCC & $99: 1$ \\
\hline LIPODISKS-FP & DSPC, DSPEPEG, DSPEPEGMCC & $80: 19: 1$ \\
\hline
\end{tabular}

\section{S1.1. Particle characterization (initial screening)}

The nanoparticles were characterized with regards to their physicochemical properties (Table S2). The amount of fusion protein coupled per particle was estimated from the total protein content determined using a fluorometric assay and from the total particle content estimated using either NTA (liposomes) or the total phosphorus content, yielding the total phospholipid content, and geometrical considerations (lipodisks). In addition to the surface-bound fusion protein, the POPC-PE-FP and POPC-PEG-FP liposomes also had the fusion protein encapsulated. There was on average 290 and 140 CTA1-3Ea-DD/particle per POPC-PEG-FP and POPC-PE-FP, respectively, of which 21 and 13\% were encapsulated (Table S2). However, it was not trivial to reproducibly control the level of encapsulation, which varied by almost a factor 3 between different 
batches (data not shown). In order to attempt to elucidate the role of fusion protein load, while ruling out any effect of the ratio of surface-bound to encapsulated fusion protein, POPC-high-FP and POPC-low-FP were formulated with only surface-bound fusion protein. These formulations carried on average 100 and 30 CTA1-Ea-DD/particle, respectively. The DSPC-PEG-FP* and the Lipodisks-FP, also with only surface-bound fusion protein, had 290 and 6 CTA1-3Ea-DD/particle, respectively.

Table S2. Summary of the physicochemical characterization of the different lipid particle formulations used for the initial screening. The zeta potentials of POPC-high-FP and POPC-low-FP were not measured.

\begin{tabular}{|c|c|c|c|c|c|c|}
\hline & $\begin{array}{l}\text { POPC-PE- } \\
\text { FP }\end{array}$ & $\begin{array}{l}\text { POPC-PEG- } \\
\text { FP }\end{array}$ & $\begin{array}{l}\text { POPC- } \\
\text { HIGH-FP }\end{array}$ & $\begin{array}{l}\text { POPC- } \\
\text { LOW-FP }\end{array}$ & $\begin{array}{l}\text { DSPC-PEG- } \\
\text { FP* }\end{array}$ & $\begin{array}{l}\text { LIPODISKS- } \\
\text { FP }\end{array}$ \\
\hline PROTEIN TYPE & $\begin{array}{l}\text { CTA1-3Ea- } \\
\text { DD }\end{array}$ & $\begin{array}{l}\text { CTA1-3Ea- } \\
\text { DD }\end{array}$ & $\begin{array}{l}\text { CTA1-Ea- } \\
\text { DD }\end{array}$ & $\begin{array}{l}\text { CTA1-Ea- } \\
\text { DD }\end{array}$ & $\begin{array}{l}\text { CTA1-3Ea- } \\
\text { DD }\end{array}$ & $\begin{array}{l}\text { CTA1-3Ea- } \\
\text { DD }\end{array}$ \\
\hline $\begin{array}{l}\text { \# } \\
\text { PROTEINS/PARTICLE }\end{array}$ & 140 & 290 & 100 & 30 & 290 & 6 \\
\hline $\begin{array}{l}\text { ENCAPSULATED } \\
\text { PROTEIN }(\%)\end{array}$ & 13 & 21 & 0 & 0 & 0 & 0 \\
\hline $\begin{array}{l}\text { DIAMETER (MEAN, } \\
\text { NM) }\end{array}$ & 120 & 150 & 110 & 110 & 110 & 22 \\
\hline $\begin{array}{l}\text { ZETA POTENTIAL } \\
(\mathrm{MV}, \pm \mathrm{SD})\end{array}$ & $-22.8 \pm 1.8$ & $-7.1 \pm 0.7$ & - & - & $-13.3 \pm 0.4$ & $-8.6 \pm 0.5$ \\
\hline $\begin{array}{l}\text { PEG-LIPID CONTENT } \\
(\%)\end{array}$ & 0 & 1 & 0 & 0 & 1 & 20 \\
\hline PHASE & $\begin{array}{l}\text { liquid } \\
\text { disordered }\end{array}$ & $\begin{array}{l}\text { liquid } \\
\text { disordered }\end{array}$ & $\begin{array}{l}\text { liquid } \\
\text { disordered }\end{array}$ & $\begin{array}{l}\text { liquid } \\
\text { disordered }\end{array}$ & gel & Gel \\
\hline
\end{tabular}

All fusion protein-carrying liposomes had a mean hydrodynamic diameter between 110 and $150 \mathrm{~nm}$ (Table S2, determined with NTA) and distributions with a full width at half maximum of approximately $50 \mathrm{~nm}$ (Figure S1A-C). The Lipodisks-FP size distribution (Figure S1C,) was determined using image analysis with ImageJ of Cryo-TEM micrographs (Figure S1C, inset); the mean diameter, not including FP and PEG spacers, was $22 \mathrm{~nm}$ (Table S2).
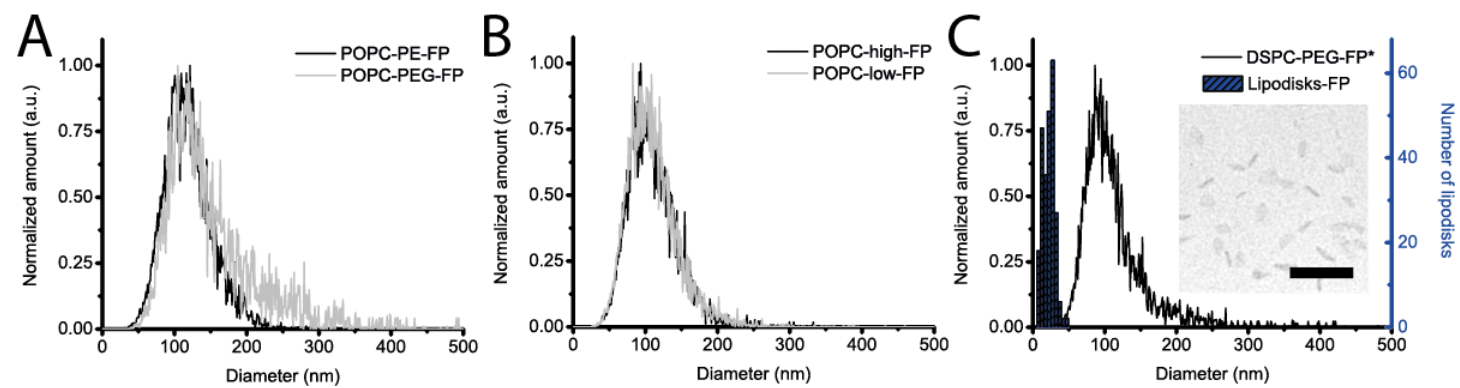

Figure S1. (A-C) Size distributions of the different particle formulations (see figure labels) presented in Table S2. NTA was used for size determination of the liposomes, while the size distribution of the lipodisks was determined from Cryo-TEM micrographs (see inset, scale bar: $100 \mathrm{~nm}$ ).

All particles had a net negative surface charge (Table S2) as determined by laser Doppler electrophoresis. The POPC-PE-FP formulation was the most negatively charged, $-22,8 \mathrm{mV}$, while the charge of all PEG-containing formulations were closer to neutral (zeta potential ranging from -7.1 to $-13.3 \mathrm{mV}$ ), which is consistent with the idea that PEG can have a charge shielding effect. ${ }^{2}$ In addition, it is noteworthy that the formulations investigated are expected to exhibit differences in the phase of their lipid membranes: DSPC-PEG-FP* and Lipodisks-FP comprise mainly DSPC, with a transition temperature of $55^{\circ} \mathrm{C}$, and are thus expected to be in the gel phase (Table S2); 
while all other formulations were mainly composed of POPC (with a transition temperature of $-2^{\circ} \mathrm{C}$ ) and $10 \%$ cholesterol, which is a composition that, similarly to the DOPC-based formulation used in the main paper, is in the liquid disordered phase at physiological temperature. ${ }^{3}$

\section{$\underline{\text { S1.2. Antigen presentation assay }}$}

The immunogenicity of the lipid formulations summarized in Table S2 was evaluated using the in vitro cell model described in the main text. To assess functional antigen presentation as well as levels of activation for the dendritic cells, the geometric mean fluorescence intensities of YAe and surface-bound MHC II were plotted against incubation time and normalized between zero at the beginning of the experiment and to unity at the value of the free fusion protein at $24 \mathrm{~h}$ (Figure S2A-C). In all cases, the main increase in antigen presentation signal occurred within the first few hours. However, it was observed that DSPC-PEG-FP* was the only formulation that gave a notable increase in antigen presentation compared to the free fusion protein at all time points. This is further illustrated in Figure 2 (main text), which shows the cumulative amount of antigen presented over the whole time frame studied.
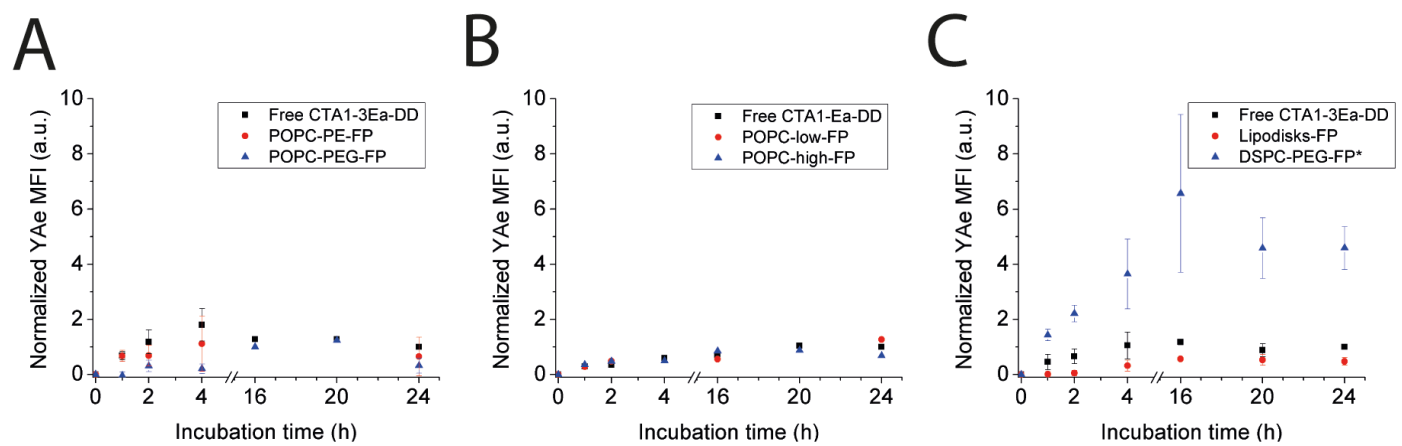

Figure S2. (A-C) YAe median fluorescence intensity as function of time as measured by flow cytometry. All error bars are standard error of the mean.

\section{$\underline{\text { S1.3 Additional experimental procedure (initial screening) }}$}

Lipid nanoparticle preparation. To produce lipodisks, a lipid film composed of DSPC, DSPEPEG and DSPEPEGMCC (80:19:1 mol\%) was rehydrated in NaAc saline to a lipid concentration of $15 \mathrm{mM}$. The rest of the preparation was done as described in the main text for gel phase liposomes. To prepare liposomes with encapsulated fusion protein (POPC-PE-FP and POPC-PEG-FP) the method described in the main paper for fluid phase liposomes was followed with the exception that $\mathrm{NaAc}$ saline $(10 \mathrm{mM}$ sodium acetate, $150 \mathrm{mM} \mathrm{NaCl}, \mathrm{pH}=5.0$ ) containing $0.16 \mathrm{mg} / \mathrm{ml} \mathrm{CTA1-3Ea-DD} \mathrm{was}$ used when rehydrating the lipid films. Unincorporated fusion protein was removed following extrusion using Amicon Ultra $100 \mathrm{kDa}$ cut-off centrifugal filters (Millipore, USA) as follows: $200 \mu \mathrm{NaAc}$ saline and $200 \mu \mathrm{l}$ lipid vesicle solution was added to each filter and centrifuged $\left(5 \mathrm{~min}, 8000 \mathrm{~g}, 10^{\circ} \mathrm{C}\right.$ ) followed by a further dilution with $200 \mu 1 \mathrm{NaAc}$ saline in each filter and another centrifugation $\left(5 \mathrm{~min}, 8000 \mathrm{~g}, 10^{\circ} \mathrm{C}\right)$. The solution was recovered by inverting the filters and centrifuging $\left(1 \mathrm{~min}, 8000 \mathrm{~g}, 10^{\circ} \mathrm{C}\right)$. Fusion protein was then coupled to the surface of liposomes as described in the main paper. To produce POPC-low-FP, the fusion protein was diluted $3 \mathrm{X}$ prior to coupling. 
Cryogenic Transmission Electron Microscopy. Cryogenic Transmission Electron Microscopy (Cryo-TEM) was used to confirm formation of lipodisks and to characterize their size. Samples were prepared by depositing a lipodisk-containing droplet on a copper grid covered with a holey polymer film. The grid was blotted with filter paper at $25^{\circ} \mathrm{C}$ and close to $100 \%$ relative humidity in a custom-built climate chamber in order to prepare thin liquid films. Vitrification of the films was achieved by plunging them immediately into liquid ethane at $-183^{\circ} \mathrm{C}$. The samples were kept below $-165^{\circ} \mathrm{C}$ during transfer to the microscope and throughout the TEM analysis, which was performed with a Zeiss TEM Libra 120 instrument (Carl Zeiss NTS, Germany) operated at $80 \mathrm{kV}$ in zero-loss bright-field mode. A TRS slow scan CCD camera system (TRS GmbH, Germany) and iTEM software (Olympus Soft Imaging Solutions GmbH, Germany) were used to record digital images under low dose conditions with underfocus of $1-3 \mu \mathrm{m}$ to enhance the contrast.

Lipid quantification for lipodisks. To estimate the Lipodisk-FP concentration the total phospholipid concentration was determined by phosphorus analysis, which was performed as described by Paraskova et al. with the modification that digestion was performed solely by dry ashing at $550^{\circ} \mathrm{C}$ overnight. ${ }^{4}$

Estimation of number of fusion proteins per particle. The mean number of fusion proteins per liposome was calculated from the protein content values obtained via CBQCA assay and the particle concentration measured with NTA. The number of fusion proteins per Lipodisk-FP was estimated using the measured protein and lipid concentrations in combination with geometric considerations as follows: the disks were considered circular double lipid layers with a diameter of $17.2 \mathrm{~nm}$ (the mean diameter from Cryo-Transmission Electron Microscopy with the length of two DSPE anchors, $2.35 \mathrm{~nm}$, subtracted), comprising lipids with a footprint of $0.497 \mathrm{~nm}^{2},{ }^{5}$ and the enclosing rim consisting of PEGylated lipids, making up $20 \%$ of the total number of lipids in the disk . 


\section{Section S2: Supporting Figures}

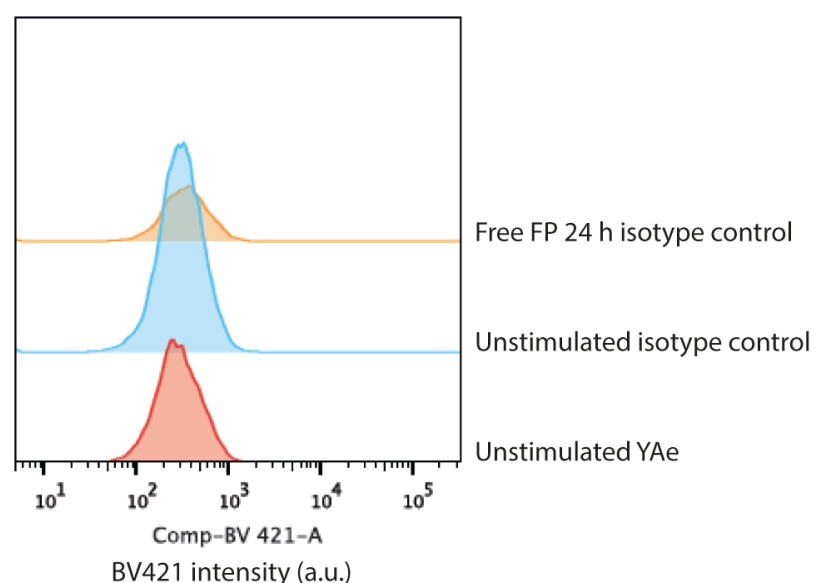

Figure S3. Controls showing that unspecific binding from isotype staining of both unstimulated and activated cells is undistinguishable to that of YAe staining of unstimulated cells.

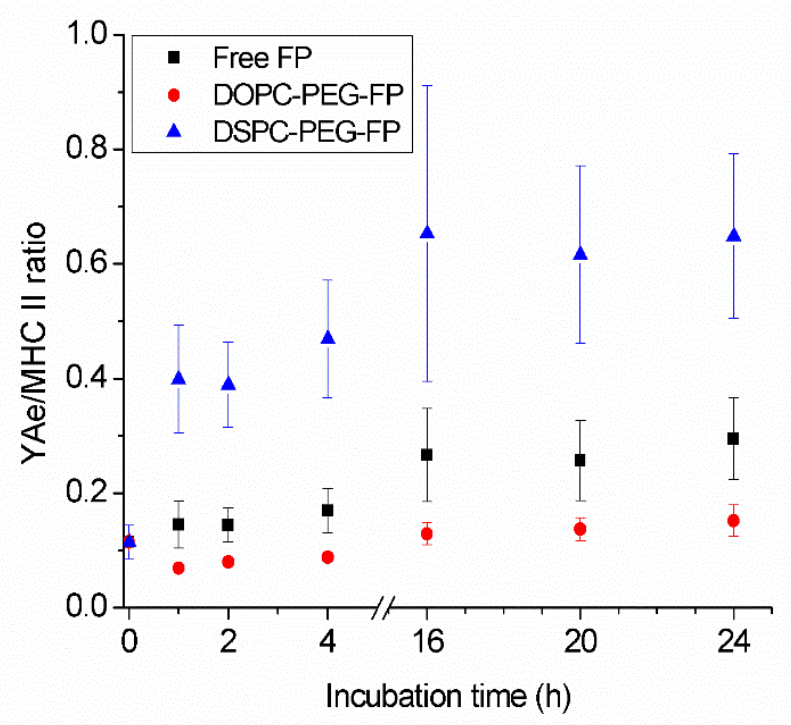

Figure S4. YAe to MHC II median fluorescence intensity over time as measured by flow cytometry. Error bars show standard error of the mean. 

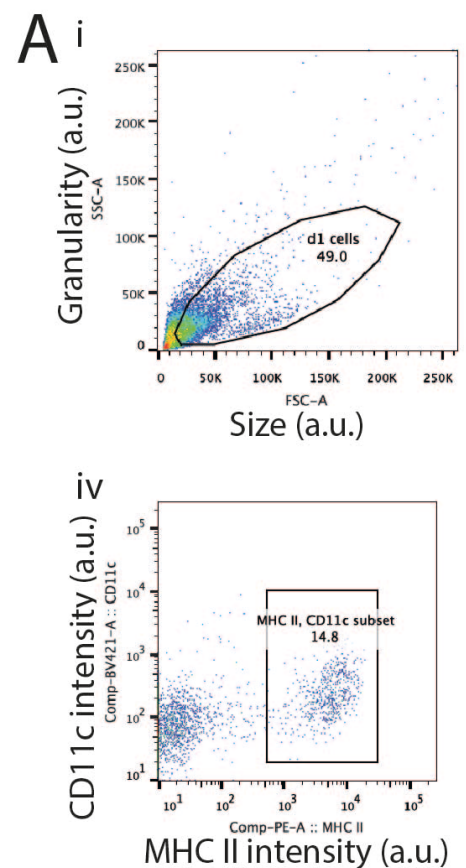

ii

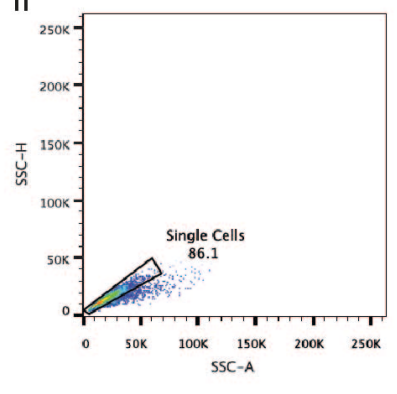

v- Unstimulated

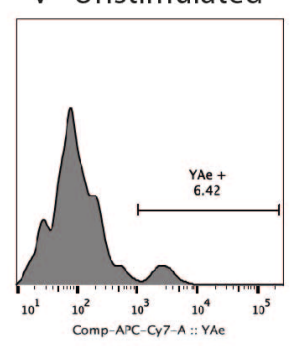

YAe intensity (a.u.)

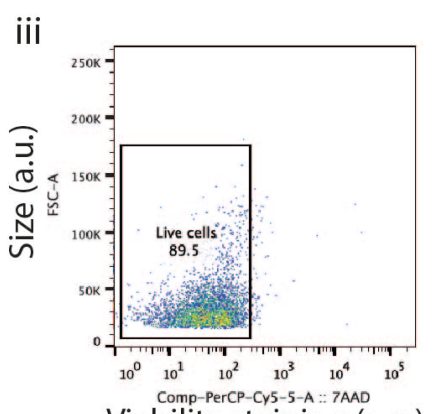

Viability staining (a.u.)

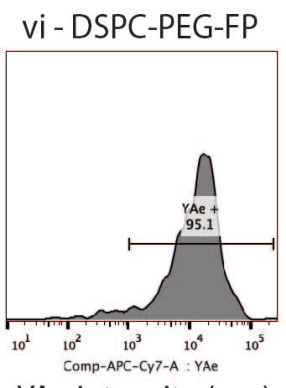

YAe intensity (a.u.)

B
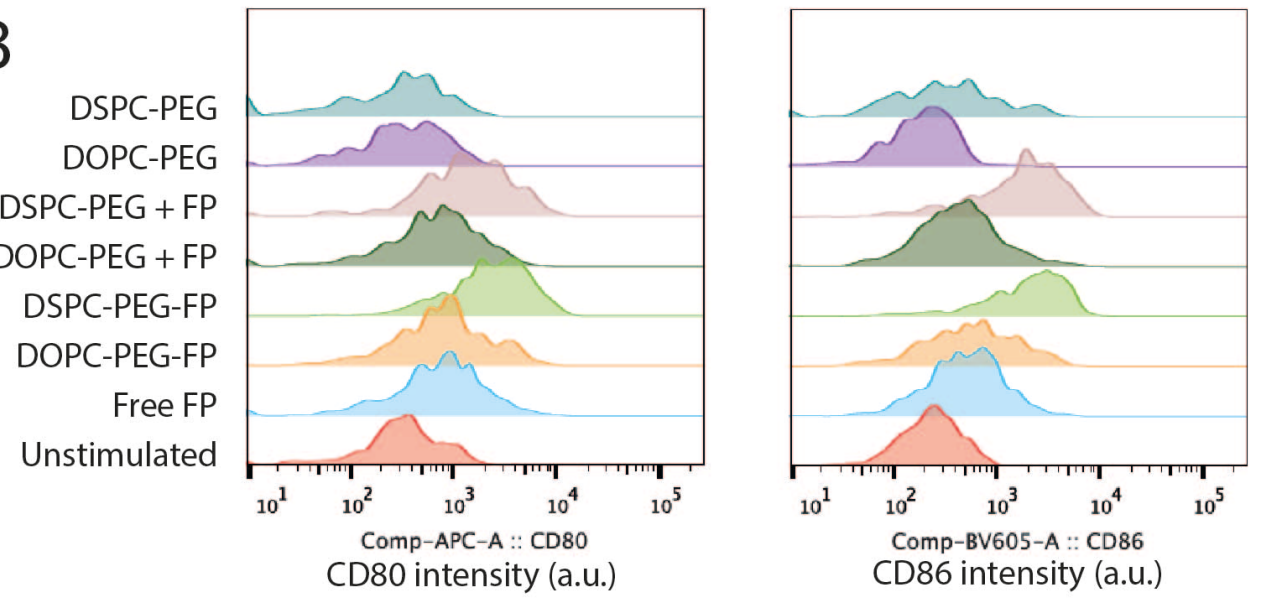

C

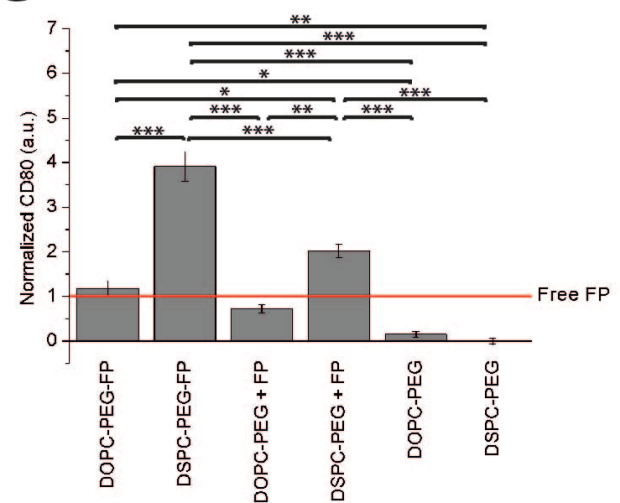

D

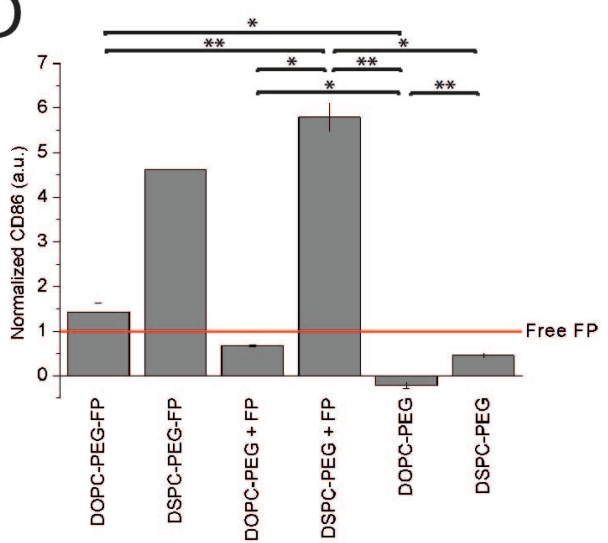

Figure S5. (A) (i-iv) Gating strategy used for quantification of costimulatory molecules and antigen presentation at 24 hours. (v) In order to only observe effects on cells that had taken up the FP, YAe+ cells were selected by placing a gate just above the main peak in the histogram of unstimulated cells. Some unspecific binding is to be expected, leading to a small number of YAe+ cells also in the unstimulated sample. (vi) As visualized by the DSPC-PEG-FP sample, the peak shifted to the right in response to stimulation, dramatically increasing the number of cells in the YAe+ gate. (B) Representative CD80 and CD86 histograms of the whole population for the different formulations. (C) Change in CD80 median fluoresence intensity at 
$24 \mathrm{~h}$ compared to free FP for the whole population. (D) Change in CD86 median fluoresence intensity at 24 h compared to free FP for the whole population. In (C) and (D) the median fluorescence intensity was normalized to zero for unstimulated cells and to unity at the value of the free fusion protein. Error bars show standard error of the mean. Statistical significance: ${ }^{*} p<0.05, * * p<0.01, * * * p<0.001$.

\section{Section S3: Supplementary References}

(1) Edwards, K.; Johnsson, M.; Karlsson, G.; Silvander, M., Effect of polyethyleneglycol-phospholipids on aggregate structure in preparations of small unilamellar liposomes. Biophys. J. 1997, 73 (1), 258-266.

(2) Oberoi, H. S.; Yorgensen, Y. M.; Morasse, A.; Evans, J. T.; Burkhart, D. J., PEG modified liposomes containing CRX-601 adjuvant in combination with methylglycol chitosan enhance the murine sublingual immune response to influenza vaccination. J. Controlled Release 2016, 223, 64-74. DOI: 10.1016/j.jconrel.2015.11.006.

(3) de Almeida, R. F. M.; Fedorov, A.; Prieto, M., Sphingomyelin/phosphatidylcholine/cholesterol phase diagram: Boundaries and composition of lipid rafts. Biophys. J. 2003, 85 (4), 2406-2416. DOI: 10.1016/s00063495(03)74664-5.

(4) Paraskova, J. V.; Rydin, E.; Sjoberg, P. J. R., Extraction and quantification of phosphorus derived from DNA and lipids in environmental samples. Talanta 2013, 115, 336-341. DOI: 10.1016/j.talanta.2013.05.042.

(5) Hartkamp, R.; Moore, T. C.; Iacovella, C. R.; Thompson, M. A.; Bulsara, P. A.; Moore, D. J.; McCabe, C., Investigating the Structure of Multicomponent Gel-Phase Lipid Bilayers. Biophys. J. 2016, 111 (4), 813-823. DOI: 10.1016/j.bpj.2016.07.016. 